\title{
Amino- and Carboxyl-terminal Sequences of a Hypocalcemic Protein Isolated from Bovine Parotid Gland
}

\author{
Akira Mizutani, Takaharu Mizutani, and Po-Feng Kuo \\ Department of Biochemistry, Faculty of Pharmaceutical Sciences, \\ Nagoya City University ${ }^{1)}$
}

(Received September 14, 1976)

\begin{abstract}
The amino-terminal sequence of a hypocalcemic protein purified from bovine parotid gland was determined as Lys-Leu- by the dansyl-Edman method in the presence of sodium dodecyl sulfate (SDS). The amount of amino-terminal lysine was quantified as 0.85 mole per mole of protein. A carboxyl-terminal sequence was analyzed by use of carboxypeptidase A and it was deduced to be-Thr-Val-Leu. Furthermore, the carboxylterminus was confirmed to be leucine alone by the hydrazinolysis method. On SDS gel electrophoresis, this protein failed to show the evidence of dissociation or association. From these results, the hypocalcemic protein was concluded to consist of a single polypeptide chain.
\end{abstract}

Keywords_- bovine parotid gland; parotin; hypocalcemic protein; dansyl-Edman method; amino-terminal sequence; carboxyl-terminal sequence; hydrazinolysis ; carboxypeptidase A

The molecular weight of the hypocalcemic protein which was isolated from bovine parotid gland was determined to be $46500 \pm 1500$ by SDS (sodium dodecyl sulfate)-polyacrylamide gel electrophoresis, ${ }^{2)}$ sedimentation equilibrium, gel chromatography on Sepharose $6 \mathrm{~B}$ in $6 \mathrm{~m}$ guanidine hydrochloride, and viscometry in $6 \mathrm{~m}$ guanidine hydrochloride. ${ }^{3)}$ This substance stimulates the lymphocytosis in neonatal mice ${ }^{4)}$ besides it possesses hypocalcemic action to rabbits.

In the present investigation, analyses of the amino- and carboxyl-terminal sequences of the hypocalcemic protein purified by the method of Mizutani, et al. ${ }^{2}$ ) were carried out in order to know whether or not the protein consists of a single polypeptide chain.

The amino-terminal sequence of the protein was elucidated by the 5-dimethylaminonaphthalene-1-sulfonyl (dansyl)-Edman method in the presence of SDS.5) On the other hand, the carboxyl-terminal sequence was analyzed using carboxypeptidase $A$ and the results obtained were confirmed by means of the hydrazinolysis method.

We also analyzed organic phosphate in the protein.

\section{Materials and Methods}

\section{Materials}

As previously reported,2) a hypocalcemic protein from bovine parotid gland was purified and the disc electropherogram of a sample is shown in Fig. 1(a).

Trifluoroacetic acid, phenylisothiocyanate, and solvents for chromatography were obtained from Wako Pure Chemical Co. Polyamide plates coated on both sides $(15 \times 15 \mathrm{~cm}$, Cheng Chin Trading Co., Taiwan) were cut to $5 \times 5 \mathrm{~cm}$. Dansyl chloride was a product of Tokyo Kasei Co., carboxypeptidase A [EC 3.4.2.1] was of Boehringer Mannheim Co., anhydrous hydrazine (95\%) was of Eastman Kodak Co., and a mineralogical lamp used for wavelength $2537 \AA$ was of Manasul Industries Co. Lysozyme [EC 3.2.1.17] was obtained from

1) Location: Tanabe-dori, Mizuho-ku, Nagoya, 467, Japan.

2) A. Mizutani, T. Kitamura, N. Yamada, H. Inaba, Y. Naito, and T. Mizutani, Chem. Pharm. Bull. (Tokyo), 22, 1955 (1974).

3) A. Mizutani, T. Mizutani, and P-F. Kuo, Chem. Pharm. Bull. (Tokyo), 24, 2733 (1976).

4) I. Suzuki, H. Inaba, Y. Naito, T. Mizutani, and A. Mizutani, J. Biochem., 78, 697 (1975).

5) A.M. Weiner, T. Platt, and K. Weber, J. Biol. Chem., 247, 3242 (1972). 


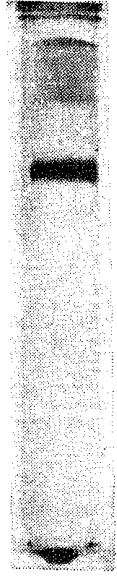

(a)

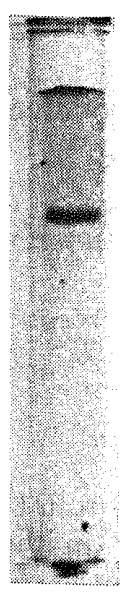

( b )
Fig. 1. Electropherograms of the Hypocalcemic Protein Isolated from the Bovine Parotid Gland

'a), disc electrophoresis of the purified substance; (b), SDS-electrophoresis of the purified substance in the presence of $4 \mathrm{M}$ urea, $1 \%$ mercaptoethanol, and $1 \%$ SDS.
Seikagaku Kogyo Co. and bovine serum albumin was from Armour Co.

Standard dansyl-amino acids were prepared according to Weiner, et al. ${ }^{5}$ )

\section{Methods}

Analysis of Amino-terminal Sequence-Amino-terminal sequence was analyzed with $1 \mathrm{mg}$ of sample by the dansylEdman method in the presence of SDS. ${ }^{5,6)}$ Ascending chromatography was performed using four solvents according to the method of Woods and Wang ${ }^{7}$ as modified by Hartley. ${ }^{6}$ ) The solvents used were as follows.

Solvent I: $1.5 \%$ formic acid in water

Solvent II: benzene-acetic acid, $9: 1, \mathrm{v} / \mathrm{v}$

Solvent III: ethyl acetate-acetic acid-methanol, 20:1: $1, \mathrm{v} / \mathrm{v} / \mathrm{v}$

Solvent IV: $\quad 0.05 \mathrm{M} \mathrm{Na}_{3} \mathrm{PO}_{4}$ in $25 \%$ aqueous ethanol Quantitative Analysis of Amino-terminal Amino AcidThe quantitative estimation of amino-terminal amino acid was performed essentially by the method of Gros and Labouesse. ${ }^{8)}$ Polyamide plates were used for thin-layer chromatography of dansyl amino acids in the present experiments. Standard bisdansyl-lysine (10 nmoles) was spotted onto the polyamide plate. The purified protein $(19.0 \mathrm{nmoles})$ was subjected to dansylation and hydrolysis, and one half of this reaction product (9.5 nmoles) was spotted onto another plate. The solvents used for chromatography were I, II, and III. The area which corresponds to the spot for bis-dansyl-1ysine was cut to a piece of $0.5 \times 0.5 \mathrm{~cm}$ and extracted with $3 \mathrm{ml}$ of a mixed solvent (chloroform-methanol-acetic acid, $7: 2: 2, \mathrm{v} / \mathrm{v} / \mathrm{v}$ ). After evaporation of the solvent, the dried extract was dissolved in $3 \mathrm{ml}$ of ethanol. The amount of dansyl-amino acid was estimated by measuring absorbance of the solution at $250 \mathrm{~nm}$ in comparison with standard bis-dansyl-lysine.

Analysis of Carboxyl-terminal Sequence by Carboxypeptidase A-Carboxyl-terminal sequence was analyzed by use of carboxypeptidase A according to the method of Frankel-Conrat, et al. ${ }^{9}$ ) The enzyme solution, which was previously treated with diisopropyl fluorophosphate, was added to the sample solution $(12 \mathrm{mg}, \mathrm{pH} 8.0)$ and the final volume was made up to $6 \mathrm{ml}$. The resulting solution was incubated at $25^{\circ}$ and $1 \mathrm{ml}$ aliquots of the solution were taken out at the time of $0,0.5,2,4,8$, and $24 \mathrm{hr}$. The digestion was stopped by adding $0.1 \mathrm{ml}$ of $1 \mathrm{~m}$ citric acid, and the amino acid fraction was obtained by gel filtration on Sephadex G-10, followed by the analysis of the amino acids using an amino acid analyzer.

Analysis of Carboxyl-terminal Amino Acid by Hydrazinolysis-Method-One mg (21 nmoles) of the hypocalcemic protein was subjected to hydrazinolysis with $0.5 \mathrm{ml}$ of anhydrous hydrazine at $60^{\circ}$ for $9 \mathrm{hr}$ in the presence of $65 \mathrm{mg}$ of hydrazine sulfate. ${ }^{10,11)}$ After drying the reaction mixture, the residue was dissolved in water and extracted with enanthaldehyde, then extraction was repeated by use of ethyl acetate to remove enanthaldehyde remained in the solution. The carboxyl-terminal amino acid liberated was dansylated, and identified with the aid of polyamide plate as described above. The blank test was performed using $65 \mathrm{mg}$ of hydrazine sulfate.

Analytical Polyacrylamide Gel Electrophoresis-Polyacrylamide gel disc electrophoresis of a sample was performed according to Davis' method. ${ }^{12}$ )

Examination of Dissociation of the Protein Molecule by SDS Gel Electrophoresis-_-SDS gel electrophoresis was performed by the method of Dunker and Rueckert ${ }^{13)}$ on $10 \%$ polyacrylamide gel in the presence of $4 \mathrm{~m}$ urea, $1 \%$ mercaptoethanol, and $1 \%$ SDS.

Determination of Phosphate in the Hypocalcemic Protein-The amount of organic phosphate in the protein was determined by the method of Miyazaki and Takemura, ${ }^{14}$ ) The sample used was $1.535 \mathrm{mg}$.

6) B.S. Hartley, Biochem. J., 119, 805 (1970).

7) K.R. Woods and K-T. Wang, Biochim. Biophys. Acta, 133, 369 (1967).

8) C. Gros and B. Labouesse, Eur. J. Biochem., 7, 463 (1969).

9) H. Frankel-Conrat, J.I. Harris, and A.L. Levy, "Methods of Biochemical Analysis," Vol. II, $1955,359$.

10) S. Akabori, K. Ohno, and K. Narita, Bull. Chem. Soc. Japan, 25, 214 (1952).

11) Y. Kawanishi, and K. Narita, Tampakushitsu Kakusan Koso, 8, 67 (1963).

12) B.J. Davis, Ann. N. Y. Acad. Sci. 121, 404 (1964).

13) A.K. Dunker and R.R. Rueckert, J. Biol. Chem., 224, 5074 (1969).

14) M. Miyazaki and S. Takemura, J. Biochem. (Tokyo), 63, 637 (1968). 


\section{Results}

\section{Amino-terminal Sequence}

The spot of bis-dansyl-lysine was detected as the amino-terminal amino acid of this sample. Dansyl-leucine appeared as the second amino acid from the terminus. The third amino acid could not be identified because several spots appeared on the plate. Thus the amino-terminal sequence of this protein was deduced to be Lys-Leu-.

\section{Quantitative Analysis of Amino-terminal Amino Acid}

The absorbance at $250 \mathrm{~nm}$ of bis-dansyl-lysine obtained from 9.5 nmoles of the purified sample contained in $3 \mathrm{ml}$ of the extract was 0.056 . The molar quantity of bis-dansyl-lysine per mole of the purified sample was estimated to be 0.64 by using the reported molecular extinction coefficient, ${ }^{8}$ and the value 0.64 was corrected as 0.85 using the yield $(75 \%)$ obtained from a control experiment with use of standard bis-dansyl-lysine.

\section{Analysis of Carboxyl-terminal Sequence with Carboxypeptidase A}

Figure 2 shows the time courses of the amino acids liberated from the protein by the action of carboxypeptidase A.

As seen from this figure, leucine was found to be the first amino acid liberated, and valine and threonine may follow as the second and third.

\section{- Analysis of Carboxy-terminal Amino Acid by Hydrazinolysis Method}

In the blank test, a spot appeared at around the position of dansyl-glycine as shown in Fig. 3(a) but its fluorescent color was blue in contrast to yellowish green of dansyl-glycine. Only dansyl-leucine spot appeared on the chromatogram of a digest of the purified sample protein. A yellowish fluorescent spot appeared at the position of dansyl-arginine and it should be attributed to an artifact, since this spot did not migrate with solvents III and IV. Consequently, the carboxyl-terminal amino acid of this protein was confirmed as leucine, as shown in Fig. 3(b), and the carboxyl-terminal sequence was deduced to be most likely -ThrVal-Leu.

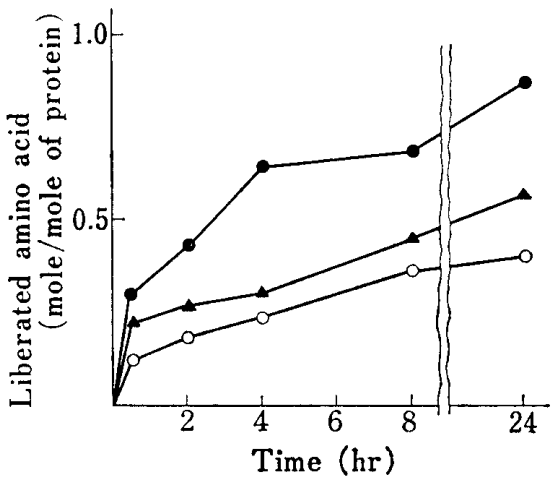

Fig. 2. Time Courses of Amino Acids Liberated from the Purified Sample with Carboxypeptidase A $\checkmark$ : leucine, $\mathbf{A}$ : valine, $\bigcirc$ : threonine
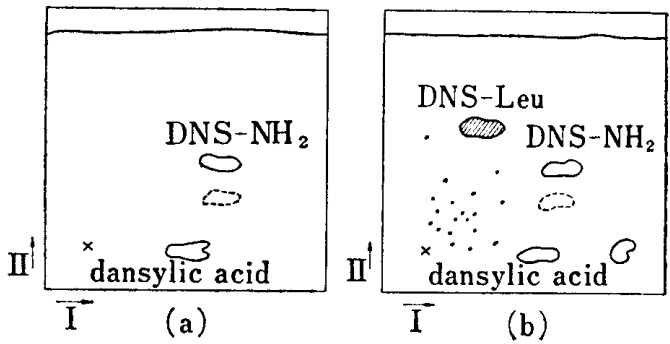

Fig. 3. Chromatograms of DNS-amino Acid Obtained by Hydrazinolysisdansylation Procedures on Polyamide Plates

(a), blank test; (b), DNS-amino acid of carboxylterminus

\section{Determination of Phosphate in the Hypocalcemic Protein}

No significant amount of organic phosphate was detected in the protein.

\section{Discussion}

The amino-terminal sequence of the hypocalcemic protein was determined as Lys-Leu-, but the 3rd amino acid was hard to characterize, since the several spots of dansyl-amino 
acids appeared on the chromatogram. According to Weiner, et al.,5) an amino-terminal sequence of 5 to 10 residues could be determined for any protein by SDS-dansyl-Edman procedures. In our preliminary experiments using both lysozyme and bovine serum albumin, spots for the 3rd dansyl-amino acids were clearly detected to be phenylalanine and histidine, respectively, in agreement with the known sequence of Lys-Val-Phe- in lysozyme and that of Asp-Thr-His- in bovine serum albumin. The failure to characterize the $3 \mathrm{rd}$ residue in the present hypocalcemic protein may be attributable to its nature from which the sample protein becomes insoluble during treatments, and thereby Edman degradation could not proceed smoothly. The protein used appears to be highly pure, because we could estimate 0.85 mole of the amino-terminal lysine per mole of the protein as its dansyl derivative.

Carboxypeptidase A liberated leucine, valine, and threonine from the protein in this order, suggesting the sequence of -Thr-Val-Leu. However, we cannot exclude a possibility that the protein consists of a subunits, one of which has leucine and the others have valine or threonine as carboxyl-termini. In order to exclude the possibility, carboxylterminal analysis by hydrazinolysis was performed, and leucine alone was detected as the carboxyl-terminal amino acid. Thus it becomes evident that both valine and threonine are not situated at the carboxyl-terminus. Meanwhile, in the studies on molecular weight determination by the sedimentation equilibrium in aqueous solution ${ }^{3)}$ and on SDS-polyacrylamide gel electrophoresis in the presence of $4 \mathrm{~m}$ urea, $1 \%$ mercaptoethanol, and $1 \% \mathrm{SDS}$, this protein failed to show any evidence of dissociation or association as shown in Fig. 1(b). Therefore, we would like to conclude that the hypocalcemic protein consists of a single polypeptide chain with a molecular weight of $46500 \pm 1500$ and has Lys-Leu- and -Thr-ValLeu at the amino- and carboxyl-terminal sequences, respectively.

When the condensation product of the hydrazides with enanthaldehyde was not removed thoroughly, the yellowish green fluorescent spot appeared on the upper area of the chromatogram with solvent I, but it did not migrate with solvent II. This spot was an artifact, since none of the usual dansyl-amino acids corresponded to this spot. In blank test, a spot appeared at around the position of dansyl-glycine. If the carboxyl- terminus of the position was a glycine, it must be difficult to distinguish between glycine and the artifact which locate at the position of dansyl-glycine on the chromatogram. The difficulty in such a case may be resolved by increasing the amount of sample to be analyzed and measuring the absorption spectrum of the dansyl derivative extracted.

The use of the dansyl technique for the characterization of amino acid released by the hydrazinolysis was reported previously by Mesrob and Holeyšovský, ${ }^{15}$ ) and Nedkov and Genov. ${ }^{16)}$ They used benzaldehyde to remove resulting amino acid hydrazides in the hydrazinolyzate, but we used enanthaldehyde. This method was done in less amounts of artifacts on the polyamide plate compared to that in the benzaldehyde treatment. The results agreed with that of Kusama who used serum albumins. ${ }^{17)}$

Acknowledgement The authors are grateful to Dr. Hideo Goto for amino acid analysis and Miss Keiko Inagaki for technical assistance.

15) B. Mesrob and V. Holeyšovský, Collection Czech. Chem. Commun., 32, 1976 (1967).

16) P. Nedkov and N. Genov, Biochim. Biophys. Acta, 127, 544 (1966).

17) K. Kusama, J. Biochem. (Tokyo), 44, 375 (1957). 